Ciencia y Educación, Vol. 4, No. 2, mayo-agosto, 2020

ISSN (impreso): 2613-8794・ISSN (en línea): 2613-8808

DOI: https://doi.org/10.22206/cyed.2020.v4i2.pp117-125

\title{
Educación por competencias en República Dominicana: perspectiva crítica sobre la práctica
}

\author{
Competency-based education in the Dominican Republic: \\ a critical perspective on practice
}

Edwin Santana Soriano ${ }^{a}$ ORCID: 0000-0002-4314-6531

Recibido: 1/02/2020 • Aprobado: 13/03/2020

Cómo citar: Santana Soriano, E. (2020). Educación por competencias en República Dominicana: perspectiva crítica sobre la práctica. Ciencia y Educación, 4(2), 117-125. Doi: https://doi.org/10.22206/cyed.2020.v4i2.pp117-125

\section{Resumen}

Se intenta llamar la atención sobre algunas de las dificultades que se enfrentan a la hora de implementar el modelo de educación por competencias en el sistema educativo formal dominicano. Se hace un esbozo de las diferencias entre el denominado "modelo tradicional" y el modelo de educación por competencias para comparar la teoría con la práctica actual, presentar el enfrentamiento entre el deber ser y el ser en lo que atañe a la aplicación práctica del modelo en la República Dominicana, y poner en relieve algunas trabas para la instauración cabal del enfoque en lo que respecta a las exigencias que hace el modelo tanto al alumnado como al profesorado, frente a realidades y actitudes de ambos actores en el contexto dominicano, revelando limitaciones y vacíos que deben tenerse en cuenta para la adopción de políticas educativas que permitan su superación y lograr una ejecución fructífera del modelo.

Palabras clave: desarrollo de habilidades; actitud del estudiante; actitud del docente; evaluación escolar; República Dominicana.

\begin{abstract}
An attempt is made to draw attention to some of the difficulties they face when implementing the competency-based education model in the Dominican formal education system. The differences between the so-called "traditional model" and the competency-based education model are outlined in order to compare the theory with current practice, present the confrontation between the duty to be and the being as regards the practical application of the model. in the Dominican Republic, and highlight some obstacles to the full establishment of the approach regarding the demands that the model makes to both students and teachers, facing realities and attitudes of both actors in the Dominican context, revealing limitations and gaps that must be taken into account for the adoption of educational policies that allow it to be overcome and achieve a successful implementation of the model.
\end{abstract}

Keywords: skills development; student attitude; teacher attitude; school evaluation; Dominican Republic.

a Universidad del País Vasco, España. Correo-e: esantana003@ikasle.ehu.eus 


\section{Introducción}

La educación bajo un enfoque por competencias, o simplemente "educación por competencias", se ha estado trabajando para ser adoptada como el modelo oficial y generalizado para el sistema formal dominicano de educación, de modo que el currículo educativo de este país ha sido repensado, revisado y rediseñado totalmente bajo este novedoso enfoque.

Dentro de los documentos oficiales que sirven de fundamento teórico a la introducción del nuevo modelo de educación en el sistema dominicano, el más relevante, por ser el más completo en cuanto a la sustentación teórica del modelo para el sistema del país, es el denominado Bases de la revisión y actualización curricular publicado por el Ministerio de Educación de la República Dominicana (MINERD) en el año 2016. Este texto define las competencias como "la capacidad para actuar de manera autónoma en contextos y situaciones diversas, movilizando de manera integrada conceptos, procedimientos, actitudes y valores" (Ministerio de Educación de la República Dominicana, 2016, p. 42). Se agrega en el texto, además, que cuando se habla de competencias no se hace referencia únicamente a destrezas o habilidades cognitivas ni al grado de eficiencia en la ejecución de una actividad, sino que las competencias implican un conjunto complejo que va desde motivaciones, pasando por las emociones y los afectos que son mediados culturalmente.

Sin embargo, esa definición adoptada por el MINERD podría parecer bastante general y compleja, empero, a la larga, en esencia y, por el énfasis que se hace en las acciones a llevar a cabo por las y los actores del sistema educativo, en la práctica, coincide con la definición común que se puede hallar en una revisión más o menos exhaustiva de la literatura.

Regularmente, y en consonancia con las actitudes y requerimientos del propio sistema educativo dominicano, cuando se habla de educación por competencias se hace referencia a una forma de educar que busca lograr en el estudiantado un "saber hacer" que se fundamenta sobre la utilización de un conjunto de recursos de manera eficaz (Lasnier, 2002, p. 32). En ese sentido, las competencias son entendidas como "conjuntos de conocimientos, habilidades, disposiciones y conductas que posee una persona y que le permiten la realización exitosa de una actividad" (Rodríguez, 1999, p. 2).

La adopción de este modelo de educación se hace para superar — a decir del propio documento oficial citado- el carácter "fragmentado y reduccionista" que es inherente a la enseñanza tradicional (MINERD, 2016, p. 40) y con "enseñanza tradicional" se hace referencia a aquel modelo educativo que, dicho de manera general, tiene un enfoque orientado a lograr que los educandos y las educandas manejen una serie de conceptos y conocimientos que les ayudarán a insertarse en la sociedad tanto en lo laboral como en el aspecto intelectual, al darles participación en la cultura universal mediante el acercamiento a textos diversos, a obras clásicas y tradicionales y la consecución de unos objetivos enfocados en unos contenidos elementales que, dependiendo del grado o nivel, el alumnado debe conocer.

La educación por competencias se enfrenta directamente a la denominada educación tradicional en más de un aspecto, como se podrá notar por las sucintas comparaciones que se hacen a continuación, que para nada agotan todas las posibles relaciones existentes entre ambos modelos ni pretenden ser el resultado de una consulta exhaustiva de toda la literatura, sino más bien una muestra que puede servir como punto de partida para, por un lado, comprender la intencionalidad general del cambio de enfoque en el contexto estudiado y, por otro, lograr un acercamiento a las limitaciones que aún persistan en su actual puesta en práctica.

De modo que, en el presente texto, que se fundamenta en una revisión bibliográfica básicamente, se buscará respuesta a las cuestiones de cuáles son las diferencias elementales entre el modelo a superar y el que le sustituye, cuáles exigencias actitudinales trae consigo el nuevo modelo para el alumnado y profesorado y, finalmente, cuáles vicios persisten en el sistema educativo formal dominicano que deben ser superados para una implementación fructífera del enfoque por competencias. 


\section{Educación por competencias o modelo "tradicional": el enfrentamiento}

Cuando se hacen comparaciones entre ambos modelos - el enfoque por competencias y el modelo "tradicional" - en lo que respecta al rol que debe jugar tanto el profesorado como el alumnado en el aula, básicamente esto es lo que suele encontrarse: se dice que mientras en la educación "tradicional" se trata básicamente de una relación unidireccional, en la que el o la docente, con experticia en la asignatura que imparte, lleva los conocimientos al aula y el estudiantado se muestra con receptividad a fin de lograr el aprendizaje esperado - aprendizaje que debe venir predefinido por el currículo educativo(Hernández, 2007; Royo, 2018); en la educación por competencias la participación del alumno y la alumna es mucho más activa, pues el profesor o profesora ha de mostrarse como un orientador del alumnado hacia el logro del aprendizaje esperado, aprendizaje que siempre será una o varias competencias —habilidades para resolver situaciones determinadas de la vida real, como ya se ha visto- y cuyos indicadores de logro, proveídos por el currículo oficial del sistema educativo en cuestión, constituyen los criterios para juzgar los avances de ese alumnado (Beresaluce, Peiró y Ramos, 2014).

En ese mismo tenor, el modelo por objetivos o "tradicional" es identificado con el conductismo en psicología (Segura, 2005), mientras que el enfoque por competencias se identifica con la tradición constructivista (Méndez y Martínez-Fernández, 2015). Lo primero porque, desde ese enfoque conductista, se presupone un determinismo de la conducta que a su vez permite "la medida objetiva, el control exterior y la predicción casi absoluta del comportamiento" (Pantoja, 2015, p. 3). Lo segundo, porque el enfoque por competencias, en tanto parte de su sustento parte de teorías constructivistas, asume que el conocimiento lo construye el sujeto a partir de conocimientos previos, por medio de la interacción social y al reconocer significatividad en los nuevos aprendizajes (Cuevas, Rocha, Casco y Martínez, 2011).

Por otro lado, en lo que respecta a los contenidos, se arguye frecuentemente que una diferencia importante entre ambos enfoques es que, en lugar de prestar especial atención a los contenidos a ser impartidos en la docencia como se hacía tradicionalmente, en el modelo por competencias los contenidos no revisten tanta importancia en comparación con la que revisten los objetivos de aprendizaje, constituyendo así los contenidos solo un medio para alcanzar un fin (Díaz, 2006).

Finalmente, en lo que atañe a la evaluación, esta también representa un punto de comparación y debate cuando se confrontan ambos modelos, pues, como propugnan especialmente los detractores de la educación por objetivos o "tradicional", la evaluación juega un papel totalmente distinto en los dos enfoques: se dice que mientras en la educación tradicional la evaluación sirve para demostrarle al alumno cuánto le falta aprender para estar a la altura que espera el sistema respecto a un conocimiento específico (Mejía, 2012), en la educación por competencias la evaluación constituye una herramienta de información importante para que el profesor-orientador se entere de qué tanto se está aprovechando su guía respecto a los objetivos que quieren lograrse en cada uno de los estudiantes (Barrón, 2005).

Desde esa perspectiva, la evaluación en la educación por competencias, contrario a como se dice sucede en el modelo tradicional, tiene una función más formativa que sumativa, es decir, que los resultados de las evaluaciones más que proveer una puntuación para calificar a los estudiantes, proporciona información de cuáles aspectos deben ser fortalecidos en cada una de las personas participantes para que se pueda lograr la competencia esperada en cada caso.

Sin embargo, cuando se revisa la literatura en la que se fundamenta la evaluación en el modelo "tradicional" o enfocado en objetivos, puede verse que también allí existe la concepción de una evaluación diagnóstica para que el docente sepa con seguridad dónde hacer énfasis durante el desarrollo de la docencia para el logro de los objetivos propuestos, así como de una evaluación formativa que da cuenta de cómo va el proceso, además de la evaluación sumativa que es la única que se le reconoce desde la crítica.

Una revisión de esos fundamentos de la evaluación en la educación "tradicional" puede hallarse en un trabajo de Jiménez Gómez, realizado en el año 1992, 
y en el que recoge la perspectiva teórica que sustentaba la evaluación en la educación tradicional hasta ese momento (Jiménez, 1992).

A su vez, cuando se critica el aspecto de la evaluación del enfoque por competencias, se dice que "invierte los objetivos de la enseńanza, al supeditarlos a los de la evaluación, convirtiéndose ésta en el criterio principal del aprendizaje, y no éste en criterio de la evaluación” (Del Rey y Sánchez-Parga, 2011, p. 235).

\section{Situación del modelo por competencias en la República Dominicana: crítica y práctica}

La crítica. Desde el ámbito local, la educación por competencias ha sido criticada también. La mayoría de trabajos que cuestionan la idoneidad del modelo y su aplicación en la República Dominicana proviene de profesionales de las ciencias humanas que ven en la educación por competencias una intención de manipulación y adiestramiento de las mayorías para que puedan convertirse en mano de obra especializada para la industria. En esto concuerdan con Del Rey y Sánchez-Parga (2011), para quienes se trata de un proceso que tiende a poner al estudiante al servicio de la economía, el mercado y sus necesidades, en lugar de poner a la educación al servicio del estudiante. Estos autores afirman que "se trata de reducir la educación a la fabricación de un alumno económicamente "performante"; adiestrado para ser competitivo en los mercados profesionales y del trabajo" (Del Rey y Sánchez-Parga, 2011, p. 235). De igual modo, coinciden con Marcuse, citado por Santana-Soriano, 2017, quien afirma que el alemán, en El hombre unidimensional, revela "la venda que tiene, sobre los seres humanos que la componen, la sociedad industrializada a fin de que ninguno sea capaz de promover y concretar una rebelión que dé al traste con un cambio radical en el sistema" (Santana-Soriano, 2017, p. 120).

Por su lado, advierte Tobón (2005) que, no obstante haberse logrado significativos avances en la conceptualización de las competencias y de las ingentes publicaciones al respecto, en la práctica persisten algunos vacíos que dificultan el empleo del enfoque en los diversos sistemas educativos, entre los que cita el hecho de que se prioriza la búsqueda de la eficacia al servicio de intereses del sistema económico imperante.

A ese respecto, la propia fundamentación teórica que se utiliza para la adopción del modelo en nuestro país, termina concediéndole crédito a las críticas cuando, en la citada Bases de la revisión y actualización curricular, al justificar el uso del enfoque por competencias, remite al Plan decenal de educación 2008-2018 y este, a su vez, dice de manera textual que, dado que el nivel de ingreso es bajo en el país, hay que hacer énfasis en la educación media y concentrar allí la atención, y que "esa concentración en la Educación Media se explica por la necesidad de disponer de personas calificadas que puedan integrarse al mercado de trabajo con salarios competitivos" (Secretaría de Estado de Educación, 2008, p. 17).

Algunos atribuyen la adopción del enfoque por competencias en el país, más que a una necesidad de cambio de modelo por demanda de los nuevos tiempos, a una agenda extranjera impuesta por compromisos adquiridos, que es el caso de Mella (2017), quien va más allá y afirma que esta no es la parte más problemática de la aplicación del modelo por competencias en el país o en cualquier parte del mundo, sino el hecho de que en su aplicación y por la forma en que se lleva a cabo, parece negar lo que en teoría propone, pues "lo problemático de este enfoque radica en (...) su modo de operar avasallador y su alto potencial estandarizado [cuya] complejidad no favorece la personalización; somete a un proceso burocrático bastante complicado, sobre todo en el momento de evaluar los aprendizajes".

Sobre la agenda "impuesta", se trata de la denominada Agenda 2030 para el Desarrollo Sostenible elaborada por la Organización de las Naciones Unidas (ONU) y cuyos objetivos propuestos a las naciones constituyen, en sus palabras, un "plan de acción a favor de las personas, el planeta y la prosperidad, que también tiene la intención de fortalecer la paz universal y el acceso a la justicia” (Organización de las Naciones Unidas, 2015). Recientemente se publicó un estudio de Murillo (2019), en el que se ponderan los "avances y retrocesos” de la República Dominicana y Colombia con miras a la consecución de los objetivos de esa 
agenda, así como las posibilidades de su aplicación en los contextos dominicano y colombiano, respectivamente.

La práctica. Antes de entrar en detalles en lo que atañe a la praxis educativa en República Dominicana, es preciso tener presente que, bajo el enfoque por competencias, se busca que cada alumno o alumna pueda alcanzar, en la medida de sus potencialidades, en el mayor grado posible las competencias deseadas para cada caso en cada asignatura. Para ello, tal como afirma Expósito (2016), es necesario que el docente tenga la posibilidad de hacer un seguimiento de cada caso particular para poder orientar donde sea necesario y fortalecer las áreas de debilidades de su alumnado para facilitarles el logro de la competencia.

De acuerdo con el portal oficial educando.edu, el sistema educativo dominicano contempla que un profesor trabaje en aula unas 33 horas a la semana de las 40 que debe permanecer en el centro, dejando siete horas para el trabajo de planificación, organización del trabajo, corrección y seguimiento de las evaluaciones, entre otros asuntos propios de la labor docente (educando.edu.do, 2014). Además, en el mismo portal, se habla de unas cuatro horas adicionales para cursos optativos.

Teniendo esta información en cuenta y vista la tabla que se muestra a continuación (tabla 1) tomada del Diseño Curricular Nivel Secundario del Ministerio de Educación, un docente del área de Lengua Española del primer ciclo de secundaria, por ejemplo, para cumplir con las horas en aula que le exige el sistema, deberá tener por lo menos unas cinco secciones (cinco secciones que, según la tabla deben tener seis horas de Lengua Española, dan un total de treinta horas). Si cada una de esas secciones tiene 30 estudiantes (que es el número aproximado de estudiantes por aula que se estila en la actualidad) este profesor tendrá un aproximado de 150 estudiantes a los cuales deberá dar seguimiento de manera individual para que logren las competencias que se supone deben lograr (o las que puedan lograr según sus condiciones y posibilidades singulares) en esa asignatura. El caso es similar para un profesor de matemáticas (asignatura de la que se deben impartir siete horas semanales), y mucho más crítico para las demás áreas como puede constatarse a partir de un cálculo simple con los datos proporcionados por la tabla 1 .

Tabla 1. Distribución del tiempo para el nivel secundario

\begin{tabular}{|l|l|l|l|l|}
\hline \multicolumn{2}{|l|}{ ÁREAS/GRADOS } & \multicolumn{3}{l|}{ PRIMER CICLO } \\
\hline \multicolumn{2}{|l|}{} & 1 ro. & 2do. & 3ro. \\
\hline Lengua Espańola & 6 & 6 & 6 \\
\hline Lenguas Extranjeras & Inglés & 4 & 4 & 4 \\
\cline { 2 - 5 } & Francés & 2 & 2 & 2 \\
\hline Matemática & 7 & 7 & 7 \\
\hline Ciencias Sociales & 5 & 5 & 5 \\
\hline Ciencias de la Naturaleza & 6 & 6 & 6 \\
\hline Formación Integral Humana y Religiosa & 2 & 2 & 2 \\
\hline Educación Física & 2 & 2 & 2 \\
\hline Educación Artística & 2 & 2 & 2 \\
\hline Cursos Optativos & 4 & 4 & 4 \\
\hline Total de horas/semanas & 40 & 40 & 40 \\
\hline
\end{tabular}

Fuente: Diseño Curricular Nivel Secundario. MINERD, 2016.

Cuando se revisan los Registros de grado, documentos en los que, entre otras cosas, se lleva el control de asistencia y calificaciones de los estudiantes, se puede notar que todos los libros de registro, de todos los niveles, contienen espacio para la inscripción de 40 alumnos y alumnas por aula. Esto no indica que sea el caso, que siempre se coloquen 40 estudiantes en una misma sección; sin embargo, una cantidad de 30 estudiantes por aulas, como actualmente se estila, es bastante difícil de manejar — por no decir imposible - si se quiere que cada uno de ellos logre las competencias para las que tiene potencial de manera individual; mucho más lo sería si ese número de espacios destinado a nombres de estudiantes en los registros se tomara como regla para la inclusión de estudiantes en un aula.

\section{Retos para la implementación cabal de modelo}

Como ya se ha visto, la educación por competencias, en vista de que requiere que el profesor mantenga una actitud de enfoque individual en la orientación 
de que los participantes puedan alcanzar, en la medida de sus propias posibilidades particulares unas competencias prefijadas (Expósito, 2016), por vía de consecuencia amerita de unas actitudes específicas también por parte del estudiante, a saber:

a) Motivación. El estudiante debe estar motivado a alcanzar las competencias prefijadas. Y esto implica otra misión para los docentes: debe ser motivador de los estudiantes frente a cada competencia. La motivación está, por razones obvias, estrechamente ligada a lo que se ha denominado como "la significatividad del aprendizaje", esto es, que "el nuevo conocimiento debe haberse relacionado con conocimientos previos, o debe estar en posibilidad de hacerlo con conocimientos ulteriores" (Saravia, 2014, p. 63), además de la consciencia de qué tan útil es o puede ser el conocimiento a obtener desde la perspectiva del estudiante.

b) Autogestión. El estudiantado, en tanto solo recibe "orientaciones" por parte del profesor o profesora y está 'motivado' por lo “significativo" del aprendizaje a obtener, tiene la responsabilidad de realizar las indagaciones necesarias para aumentar sus conocimientos sobre los contenidos esbozados en clases a fin de alcanzar de la manera más óptima los objetivos prefijados y, además, para enriquecer la interacción en clases. Acá hay que tener en cuenta que, aunque se habla en singular a fin de generalizar los enunciados, en la práctica, el alumnado recibe el 'acompañamiento' de varios profesores y profesoras simultáneamente, por lo que esa autogestión debe hacerse para cada una de las asignaturas con sus respectivos contenidos y competencias a lograr.

c) Compromiso. El estudiantado debe estar de acuerdo con que lo que se le va a presentar en clases le es significativo y consecuentemente hacerse responsable de coadyuvar en el proceso al profesor o la profesora, evitando caer, o hacer caer a sus compañeros de aulas, en distracciones de cualquier tipo que entorpezcan el desarrollo óptimo del proceso y el mejor logro de las competencias propuestas de cara a la capacidad de cada participante.
Además, a nivel sistémico, la educación por competencias requiere la posibilidad de que el profesor dé seguimiento a cada caso bajo su responsabilidad, de manera que, tras cada evaluación, sea capaz de orientar de manera individual a los estudiantes, en el entendido de que las necesidades cognitivas y actitudinales de cada persona son, por naturaleza, distintas, y esto conduce a que, por un lado, todo el alumnado no pueda adquirir las mismas competencias al mismo tiempo, y por otro, que aun los que logren adquirir las mismas competencias, no las alcancen en el mismo grado (Mano y Moro, 2009).

Esa posibilidad de seguimiento debe verse reflejada en ciertas condiciones laborales, dentro de las que se pueden citar, por considerarse esenciales, una cantidad bastante limitada de estudiantes por aula —además de la asistencia de un profesor ayudante en caso de ser necesario- y un tiempo idóneo para poder desarrollar todas las orientaciones necesarias en cada sesión de clases, de manera que todos y todas, o un porcentaje importante del estudiantado, alcance los objetivos propuestos para cada encuentro. Condiciones estas que, como se ha visto ya, en el contexto dominicano no están dadas.

Por otro lado, la característica esencial de la evaluación en el modelo por competencias, es decir, el hecho de que su principal función sea formativa, que busque dar información al profesor de los aspectos que debe reforzar en su grupo y con cada participante con miras al logro de los objetivos, obliga también al docente a mantenerse en constante modificación de su planificación diaria, pues será cada vez que evalúe que se dará cuenta de los puntos que deberá reforzar en los encuentros posteriores y para cuáles alumnos y alumnas de manera específica deberá trabajar tal o cual tema o usar cierta estrategia para ayudarlo al logro de la competencia. De modo que, aunque el docente tenga una planificación general de la docencia con estrategias de enseñanza-aprendizaje, con instrumentos de evaluación previamente concebidos y actividades pensadas para motivar y acercar al alumnado a las competencias requeridas, deberá dedicarle tiempo para la revisión de su plan, previo a cada encuentro nuevo y a la luz de los resultados que arrojen las últimas evaluaciones. 


\section{Conclusiones}

En el contexto actual de la educación preuniversitaria dominicana el enfoque por competencias, no se está llevando a cabo a plenitud, e incluso, su proyecto de aplicación debe tratarse como un plan a largo plazo que tome en cuenta realidades muy propias de este contexto para adaptar el modelo o cambiar las circunstancias, según sea posible.

Por lo pronto, se hace menester contar con una cantidad suficiente de docentes, reducir su carga académica y tener en cuenta, para la elaboración de los horarios, el tiempo que necesitará cada docente para la preparación de cada encuentro.

En cuanto al estudiante, el reto es mucho más difícil de superar, pues aquí sí se pone de manifiesto la influencia de la tradición. Ante docentes que solo atienden a la función sumativa de la evaluación, el estudiantado se habitua a jugar un rol pasivo en su proceso de aprendizaje. Esto trae consigo complicaciones a la hora de implementar el modelo por competencias que, como se ha visto, exige un alto grado de motivación intrínseca y compromiso para una autogestión óptima. No obstante, un problema actitudinal de este tipo puede verse como temporal, pues en la medida en que se arraigue la educación por competencia y pasen los años, los estudiantes que en el presente están en aulas irán saliendo y los nuevos les sustituirán ya "amoldados" al nuevo modelo; sin embargo, un cambio de actitud de carácter mediato no resultará tan fácil de llevar a cabo si se tiene en cuenta el hecho de que el sistema educativo se enfrenta a dos obstáculos importantes para realizar un cambio de paradigma y una implementación plena del modelo bajo competencias en lo que respecta a los profesores: el cambio en la logística (carga académica y límite de matrícula) y la resistencia al cambio que, ligada a la tradición, lleva a reproducir y fomentar aquellas actitudes de manera tácita e inconsciente en muchos de los casos.

En lo que respecta a la discusión de si es necesario implementar un nuevo modelo por considerar al anterior como obsoleto o poco productivo frente a los avances sociales y tecnológicos actuales, puede notarse que se ha incurrido en el error de criticar los fundamentos teóricos cuando lo que ha estado mal es la práctica. Se concluye esto porque una revisión de los fundamentos teóricos de aquel modelo que se quiere 'superar', da cuenta de principios importantes que se encuentran en los fundamentos del nuevo modelo que viene a sustituirlo y que son presentados como una innovación de este enfoque, como es el caso del papel de la evaluación, así como de otros aspectos importantes. De modo que no había que recurrir necesariamente a un cambio de modelo, sino lograr encausar las acciones hacia la idea sobre la que estas se sustentan.

Teniendo esto en cuenta, puede predecirse que no importa lo bien elaboradas que estén las teorías fundamentales de cualquier enfoque, si no se presta atención especial a la acción, se repetirá el modelo que más acomode a cada actor que tenga la libertad y posibilidad de acomodarse. Es decir, que, aunque le llamemos "enfoque por competencias" es muy probable que el cuerpo docente continúe con sus estilos de docencia que han aprendido por años si no son fiscalizados e incentivados para realizar un cambio real en su práctica.

En cuanto al enfrentamiento entre ambos modelos, el "tradicional" y "por competencias", es importante aclarar que se hace menester manejarse con mucho cuidado, pues lo nuevo, a pesar de que puede hallar resistencia en los sujetos que habrán de someterse a cambios, también puede suscitar, por un lado, una cantidad ingente de defensores cuyos trabajos, textos y presentaciones en defensa de lo nuevo eclipsen lo que pueda decirse en favor de "lo viejo"; por el otro, un temor implícito a ser tildado de "conservador", "retrógrado" o "renuente al cambio", que puede llevar a algunos que tienen algo que decir en defensa de "lo tradicional" a guardar silencio.

Por otra parte, y tras la constatación empírica, se puede observar que aquella gran cantidad de trabajos en defensa de la educación por competencias frente a la educación tradicional suele enfocarse, a la hora de definir la segunda, en una serie de características que ninguno de los que han sido educados bajo aquel modelo podría testificar que lo vivió; sin embargo, eso es tema para una investigación muy profunda y aparte, que se puede llevar a cabo más adelante. Lo 
que es evidente es que no está del todo claro el hecho de que la denominada "educación tradicional" pueda ser definida como un evento o ente lo suficientemente delimitado apoyándose en la literatura que abunda, luego del auge que ha tomado el enfoque por competencias como la solución a los problemas en la educación de cara a los nuevos tiempos.

\section{Referencias}

Barrón T., M. C. (2005). Criterios para la evaluación de competencias en el aula: una experiencia mexicana. Perspectiva Educacional, Formación de Profesores, I(45), 104-121.

Beresaluce, B., Peiró, S., y Ramos, C. (2014). El profesor como guía-orientador. Un modelo docente. Universidad de Alicante, 1-14. Recuperado de Universitar d'Alacant: https://web.ua.es/va/ice/ jornadas-redes-2014/documentos/comunicacions-posters/tema-2/392803.pdf

Cuevas, L., Rocha, V., Casco, R., y Martínez, M. (2011). Punto de encuentro entre constructivismo y competencias. AAPAUNAM Academia, Ciencia y Cultura, 5-8.

del Rey, A. y Sánchez, J. (2011). Crítica de la educación por competencias. Universitas. Revista de ciencias sociales y humanas, 233-246.

Díaz, Á. (2006). El enfoque de competencias en la educación: ¿Una alternativa o un disfraz de cambio? Perfiles educativos, 28(111), 7-36.

Educando.edu.do. (2014). El portal de la educación dominicana. Recuperado de: educando.edu. do: http://www.educando.edu.do/institucional/ noticias/en-los-centros-de-jornadas-extendidas-los-profesores-y-estudiantes-tendran-todo-su-tiempo-ocupado/

Expósito, J. (2016). El profesor como orientador. En S. N. Martín, Competencias del profesional docente (pp. 93-119). Madrid: Dykinson.

Hernández, M. G. (2007). Práctica docente y procesos comunicacionales. Revista Tecnología y Comunicación Educativa, 21(44).

Jiménez, F. (1992). Evaluación educativa tradicional. Salamanca: Universidad de Salamanca.

Lasnier, F. (2002). Réussir la formation par competences. Montreal: Guérin.
Mano, M. D. y Moro, M. (2009). La evaluación por competencias: propuesta de un sistema de medida para el grado en Información y Documentación. BiD: textos universitaris de biblioteconomia i documentació, 1(23).

Mejía, O. (2012). De la evaluación tradicional a una nueva evaluación basada en competencias. Revista electrónica Educare, 16(1), 26-46.

Mella, P. (2017). Amigo del hogar. Enfoques de competencias y educación dominicana. Recuperado de https:/www.amigodelhogar.net/2017/09/enfoques-de-competencias-y-educacion.html

Méndez, L. M., y Martínez, J. R. (2015). Modelo por objetivos Vs. modelo por competencias. Un análisis de las estrategias de aprendizaje. 3er. Congreso Internacional Multidisciplinar de Investigación Educativa (pp. 1-8). Lleida: Universidad de LLeida.

Ministerio de Educación de la República Dominicana. (2016). Bases de la revisión y actualización curricular. Santo Domingo: MINERD.

Murillo, J. (2019). Discusión de la aplicabilidad del desarrollo sostenible desde las políticas públicas en la República Dominicana y en Colombia según la adaptación educativa de la agenda 2030 . Ciencia y Sociedad, 44(2), 37-51. Doi: https:// doi.org/10.22206/cys.2019.v44i2.pp37-51

Organización de las Naciones Unidas. (2015). La Asamblea General adopta la Agenda 2030 para el Desarrollo Sostenible. Recuperado de: https://www.un.org/sustainabledevelopment/es/2015/09/la-asamblea-general-adopta-la-agenda-2030-para-el-desarrollo-sostenible/

Pantoja, L. (2015). La autorregulación (self-regulation) de la conducta y educación: teoría y técnicas. Madrid: Editorial de la Universidad Complutense de Madrid.

Rodríguez, N. (1999). La medición de las competencias. Psico Consult, 1-15.

Royo, A. (2018). Hay que ser moderno. Recuperado el 25 de noviembre de 2018, de Profesor Atticus: Educación, Política y Sociedad. Disponible en http://profesoratticus.blogspot.com/2018/11/ hay-que-ser-moderno.html 
Santana, E. (2017). Ensayos Filosóficos: reflexiones epistemológicas, ontológicas y éticas. Santo Domingo: Lulu

Saravia, J. (2014). Aprendizaje significativo y significatividad del aprendizaje. Acta Herediana, 63-76.

Secretaría de Estado de Educación. (2008). Plan Decenal de Educación 2008-2018.
Segura, M. (2005). El ambiente y la disciplina escolar desde el conductismo y el constructivismo. Revista electrónica "Actualidades Investigativas en Educación", 1-18.

Tobón, S. (2005). Formación basada en compentencias: pensamiento complejo, diseño curricular y didáctica. Bogotá: Ecoe Ediciones. 